\title{
Der Ansatz Agiles Lernen im Unternehmen
}

\author{
Gabriele Korge, Jörg Longmuß, Benjamin Höhne \\ und Agnes Bauer
}

\subsection{Sicher ist nur der Wandel}

Der aktuelle Wandel in Wirtschaft und Gesellschaft führt zu Umwälzungen in einem bislang nicht gekannten Ausmaß. Strukturen, Prozesse, Aufgabenzuschnitte verändern sich grundlegend - und dies in nahezu allen Branchen und Geschäftsbereichen. Es wird erstmalig nicht darum gehen, ein altes System aufzugeben, um in ein neues, Stabilität versprechendes System zu wechseln. Zukünftig ist von den Unternehmen und den Menschen eine neue Flexibilität gefordert, bei der es weniger um zeitlich-räumliche Verfügbarkeit geht, sondern vielmehr um ein Sich-Einlassen auf stetig wechselnde Anforderungen, Bedingungen und Situationen. Eine innovationsgetriebene Wirtschaft braucht neben der traditionellen Organisationsarchitektur agile Teams, denn in der digitalisierten Arbeitswelt wird der Mensch vor allem dort gebraucht, wo der Umgang mit unvorhergesehenen Situationen erforderlich ist. Dies erfordert einen Spagat zwischen Stabilität und Innovation, zwischen Effizienz und Flexibilität, zwischen Unternehmensvorgaben und Eigeninitiative.

G. Korge $(\triangle)$

Fraunhofer-Institut für Arbeitswirtschaft und Organisation IAO, Stuttgart, Deutschland

E-Mail: gabriele.korge@iao.fraunhofer.de

J. Longmuß

SUSTAINUM - Institut für zukunftsfähiges Wirtschaften Berlin, Berlin, Deutschland

E-Mail: j.longmuss@ sustainum.de

B. Höhne

Fernstudieninstitut, Beuth Hochschule für Technik Berlin, Berlin, Deutschland

E-Mail: bhoehne@beuth-hochschule.de

A. Bauer

ZNL TransferZentrum für Neurowissenschaften und Lernen der Universität Ulm, Ulm, Deutschland

E-Mail: agnesbauer@web.de 
Die Aufgaben vieler Menschen werden damit zunehmend von Vielfalt, Komplexität und Eigenverantwortung geprägt sein. Kollaboration und fachübergreifender Austausch bekommen eine größere Bedeutung, Arbeitsinhalte und Arbeitsweisen werden vielfältiger und unterliegen zugleich dem stetigen technischen Fortschritt.

\subsection{Die Krise der klassischen Bildungsformate}

Schon länger gelingt es immer weniger, die Menschen im Rahmen ihrer Erstausbildung oder externer Seminare so auf die Arbeit vorzubereiten, dass sie im Arbeitsalltag umfassend handlungsfähig sind. Zu kurz ist die Halbwertzeit des Wissens, zu spezialisiert sind die Unternehmen, zu situationsabhängig muss gehandelt werden, zu kontinuierlich muss gelernt werden.

Schon heute hat das Lernen in der Arbeit eine große Bedeutung, weil hier besser an den Arbeitsprozess und an konkrete Arbeitsaufgaben angeknüpft werden kann, was Voraussetzung für ein erfolgreiches Arbeitshandeln in einer komplexen Arbeitswelt ist.

Bislang greifen viele Lernkonzepte aber noch zu kurz. Aus Vorführen und Nachmachen (z. B. klassisches Einlernen), Teilnehmen (z. B. klassisches Seminar, MOOC) und Mitmachen (z. B. einfacher Qualitätszirkel, KVP-Workshop) erwächst nicht unbedingt Handlungskompetenz und zumeist auch keine Selbstreflexion. Dazu braucht es Austausch und Prozessbegleitung, mitunter auch Anleitung und Beratung. Es braucht ein Lernen, das eigene Erfahrungen in einem realen Kontext ermöglicht sowie Raum zu umfassender Reflexion gibt. Bereits in der Lernmethodik und in der Rolle der Lernbegleitung selbst müssen die Handlungsweisen und Haltungen angelegt sein, die es zu vermitteln gilt. Eigenverantwortliches agiles Arbeiten lässt sich nicht in einem fremdbestimmten Seminarumfeld erlernen.

Entsprechend sind in Zukunft vor allem Lernangebote gefragt, die im Lerngegenstand, im Lernprozess und in der Haltung der Verantwortlichen ein Abbild der Arbeitswelt der Zukunft darstellen. Das flexible Reagieren auf Veränderung ist dann wichtiger als das Festhalten an einem Curriculum oder Lernplan, die Selbststeuerung der Lernenden ist wichtiger als das Schützen vor Misserfolgen. Teamleistung zählt dann mehr als individuelle Bestleistung. Selbstreflexion zum eigenen Lernprozess und die Selbsteinschätzung persönlicher Fortschritte sind dann wichtiger als Fremdbewertungen auf Basis vorgegebener Standards.

\subsection{Das Lernen an der eigenen Arbeit orientieren}

Für eine berufliche Kompetenzentwicklung unter diesen Bedingungen müssen drei Anforderungen erfüllt sein:

- Inhaltliche Anpassbarkeit, um neue Themen möglichst schnell aufnehmen zu können; 
- Anschlussfähigkeit an vorhandene Organisationsstrukturen und Software-Infrastruktur, um mit geringem Aufwand starten zu können;

- Hohe Skalierbarkeit, um Lernangebote von wenigen Stunden bis hin zu mehreren Monaten möglich zu machen.

Bislang gibt es allerdings kaum geeignete Weiterbildungsformate, die diesen Anforderungen umfassend genügen. Klassische Formen der Qualifizierung (z. B. Seminarlehrgänge, Weiterbildungsstudiengänge) passen nicht genau genug auf die individuellen Kompetenzbedarfe und sind auch zu träge, um auf die Veränderungsdynamik in den Unternehmen zu reagieren. Sie können in der Regel nicht die angestrebte Einbettung in die unternehmerischen Prozesse gewährleisten und bieten auch keinen engen inhaltlichen Bezug zu unternehmensrelevanten Inhalten, mit dem Transferverluste minimiert werden könnten.

Als Antwort auf diese neuen Lernherausforderungen entwickelten und erprobten die Herausgeber in den letzten Jahren zusammen mit Forschungs- und Unternehmenspartnern angepasste Lernangebote, die sich methodisch bei Scrum, einem Arbeitskonzept aus der Softwareentwicklung, bedienten. Aus den gesammelten Erfahrungen und einer Zusammenführung der Konzepte entstand der Ansatz Agiles Lernen im Unternehmen. Er orientiert sich am Konzept der Lern- und Arbeitsaufgaben zur inhaltlichen Strukturierung und Anleitung des Lernens sowie an der agilen Projektmanagementmethode Scrum zur Lernorganisation. Agiles Lernen im Unternehmen greift dabei auf den vielfältigen methodischen Werkzeugkasten zurück, der für Scrum entwickelt wurde. Doch während Scrum in der Softwareentwicklung hilft, eine beherrschte Arbeitsaufgabe über einen agilen Arbeitsprozess flexibler abzuwickeln, werden im agilen Lernen die zu lösenden Aufgaben noch nicht vollständig beherrscht. Das Lernteam wird Fehler machen, und dies muss für einen produktiven Lernprozess sogar ausdrücklich möglich und akzeptiert sein. Außerdem lernt ein Lernteam nicht isoliert von seiner Arbeit - sowohl zeitlich als auch räumlich verschmelzen Lernen und Arbeiten: Das Lernen erfolgt in Etappen, in denen die Lernenden immer wieder an ihren Arbeitsplatz und in ihren Arbeitsalltag zurückkehren.

Das Lernen selbst wird strukturiert durch Lernaufgaben. Diese beschreiben, was lernend bearbeitet werden soll, und liefern Hinweise, worauf zu achten ist oder wie vorgegangen werden kann. Dadurch entfällt die klassische Rolle eines „Wissensvermittlers“ die Lernteams lernen und arbeiten selbstverantwortlich und erfahrungsorientiert.

Dieses Lernen direkt an und in der realen Praxis hat mehrere Vorteile:

- Die Teilnehmenden lernen genau das, was sie für ihre Arbeit benötigen, und nicht das, was in allgemeinen Seminaren angeboten wird. Das Gelernte ist damit direkt im Arbeitsalltag anwendbar. Dies bezieht sich nicht nur auf die Inhalte, sondern auch auf die dabei eingesetzten Arbeitsmittel.

- Die Nützlichkeit jedes Lernschritts ist unmittelbar erkennbar. Es wird nicht ,auf Vorrat" gelernt in der Hoffnung, dies eines Tages einsetzen zu können. Dies erhöht die Motivation, auch schwierige oder langwierige Aufgaben zu lösen. 
- Ausgehend von einem Lernziel, das auf Leitungsebene festgelegt wird und für den jeweiligen Lernbedarf entwickelt wurde, ist es zudem möglich, individuelle Kompetenzziele zu verfolgen. Diese Ziele verändern sich wiederum mit zunehmendem Wissen und können entsprechend angepasst werden. So lassen sich Unternehmens- und individuelle Ziele vereinbaren und stets flexibel an neue Entwicklungen anpassen.

- Das Lernthema wird zugänglicher für die Teilnehmenden, weil sie deutlich weniger Abstraktions- und Transferleistung erbringen müssen, um ihr Praxisproblem hinter der Aufgabenstellung zu erkennen. Dies erleichtert insbesondere praxisorientierten Personen den Zugang zum Thema.

\subsection{Erfahrungen mit agilen Lernprojekten}

Das Agile Lernen im Unternehmen ist als didaktisches Rahmenkonzept zu verstehen. Das heißt, orientiert am Grundkonzept erfolgt jeweils die nach Thema, Zielgruppe und Rahmenbedingungen angepasste didaktische Ausgestaltung eines spezifischen Lernangebotes, eines ,,agilen Lernprojekts“.

Die mittlerweile sehr breite Erprobung des Ansatzes Agiles Lernen im Unternehmen zeigt deutlich, dass diese Lernform auf große Akzeptanz bei den Lernenden und den Verantwortlichen stößt - nicht nur, weil das Lernen in agilen Lernprojekten Spaß macht, sondern auch, weil es durch die besondere Kombination von Lern- und Arbeitsaufgaben mit agiler (Lern)Projektorganisation gelingt, die Menschen für die Herausforderungen im Arbeitsalltag zu qualifizieren. Mit Lernaufgaben wird das erfahrungsorientierte Lernen im realen Kontext der Arbeit unterstützt und so die berufliche Handlungsfähigkeit gestärkt. Dadurch gelingt es, die Menschen auf die Arbeit der Zukunft umfassend und zielgenau vorzubereiten.

Einschränkend ist anzufügen, dass für die Durchführung von agilen Lernprojekten im Unternehmen seitens der Organisation und aller Beteiligten grundlegende Voraussetzungen zu erfüllen sind. Lernende, die die Selbststeuerung im Lernen bzw. in der Arbeit (noch) nicht gewohnt sind und auch sonst in der Vergangenheit im Lernen wenig aktiv waren, sind leicht überfordert bzw. können sich manchmal nur schwer auf den mühevolleren Weg einlassen, sich Neues eigenständig zu erarbeiten. Deshalb muss sich die Lernbegleitung auf eine lernenden-zentrierte Gestaltung einlassen. Von ihr ist eine Haltung gefordert, in der sie den Lernenden Raum zur Selbststeuerung ihres Lernens gibt und gleichzeitig Versuche der Lernenden abwehrt, die Verantwortung für das eigene Lernen abzugeben. Außerdem braucht es im organisatorischen Umfeld ein Zutrauen in das eigenverantwortliche Handeln aller Beteiligten sowie einen Freiraum zum Machen eigener Erfahrungen.

Durch die Meetings zur eigenständigen Planung des Lernens sowie zur Reflexion über Lernergebnisse (im Review) und zum Lernprozess (in der Retrospektive) muss in agilen Lernprojekten ein gewisser Mehraufwand in Kauf genommen werden. Dieser lohnt sich erst, wenn tatsächlich Kompetenzen im Sinne flexibler beruflicher Handlungsfähigkeit 
aufgebaut werden sollen. Geht es dagegen vor allem um das Aneignen von Grundlagenwissen oder um den Erwerb einfacher beruflicher Handlungsfähigkeiten zum planmäßigen Handeln in einem stabilen, definierten Umfeld, sind einfachere Maßnahmen zielführender und zeitsparender.

Im Ergebnis wird mit agilen Lernprojekten ein lösungs- und praxisorientiertes Vorgehen etabliert, bei dem sich die Teammitglieder neue Kompetenzen organisch aneignen können. Durch die wiederkehrende Reflexion und die Einbindung der Leitungsebene als Auftraggeber gelingt es darüber hinaus, einen Veränderungsprozess anzustoßen, der nachhaltig in der Organisations- und Personalentwicklung verankert werden kann.

Die Vorhaben ,inMEDIASres“ und „MeDiAL-4Q“ wurden im Frühjahr 2020 abgeschlossen. Weitere Informationen zu den Vorhaben sind zu finden unter:

https://www.dlpm.iao.fraunhofer.de/de/themen/industrie40/sprintlernen.htmlhttps:// agile-learning.eu/medial-4q/

Open Access Dieses Kapitel wird unter der Creative Commons Namensnennung - Weitergabe unter gleichen Bedingungen 4.0 International Lizenz (http://creativecommons.org/licenses/ by-sa/4.0/deed.de) veröffentlicht, welche die Nutzung, Vervielfältigung, Bearbeitung, Verbreitung und Wiedergabe in jeglichem Medium und Format erlaubt, sofern Sie den/die ursprünglichen Autor(en) und die Quelle ordnungsgemäß nennen, einen Link zur Creative Commons Lizenz beifügen und angeben, ob Änderungen vorgenommen wurden. Wenn Sie das Buch oder Teile daraus remixen, verändern oder anderweitig direkt darauf aufbauen, dürfen Sie Ihre Beiträge nur unter derselben Lizenz wie das Original verbreiten.

Die in diesem Kapitel enthaltenen Bilder und sonstiges Drittmaterial unterliegen ebenfalls der genannten Creative Commons Lizenz, sofern sich aus der Abbildungslegende nichts anderes ergibt. Sofern das betreffende Material nicht unter der genannten Creative Commons Lizenz steht und die betreffende Handlung nicht nach gesetzlichen Vorschriften erlaubt ist, ist für die oben aufgeführten Weiterverwendungen des Materials die Einwilligung des jeweiligen Rechteinhabers einzuholen.

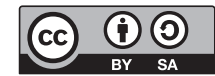

